\title{
Wnt5a stimulates chemotactic migration and chemokine production in human neutrophils
}

\author{
Young Su Jung ${ }^{1,2}$, Ha Young Lee ${ }^{1,2}$, Sang Doo Kim ${ }^{1}$, Joon Seong Park ${ }^{3}$, Jung Kuk Kim ${ }^{4}$, \\ Pann-Ghill Suh ${ }^{4}$ and Yoe-Sik Bae $e^{1,2,5}$
}

\begin{abstract}
Wnt5a is a ligand that activates the noncanonical Wnt signaling pathways ( $\beta$-catenin-independent pathways). Human neutrophils expressed several Wnt5a receptors, such as Frizzled 2, 5 and 8. Stimulation of human neutrophils with Wnt5a caused chemotactic migration and the production of two important chemokines, CXCL8 and CCL2. CCL2 production by Wnt5a was mediated by a pertussis toxin-sensitive G-protein-dependent pathway. Wnt5a also stimulated the phosphorylation of three mitogen-activated protein kinases (MAPKs: ERK, p38 MAPK and JNK) and Akt. Inhibition of ERK, p38 MAPK or JNK by specific inhibitors induced a dramatic reduction in Wnt5a-induced CCL2 production. Supernatant collected from lipopolysaccharide-stimulated macrophages induced neutrophil chemotaxis, which was significantly inhibited by anti-Wnt5a antibody. Our results suggested that Wnt5a may contribute to neutrophil recruitment, mediating the inflammation response.
\end{abstract}

Experimental \& Molecular Medicine (2013) 45, e27; doi:10.1038/emm.2013.48; published online 14 June 2013

Keywords: chemotaxis; Gi-protein coupled receptor; neutrophils; Wnt5a

\section{INTRODUCTION}

Wnts, which are secreted glycoproteins, are reported to be important in the regulation of diverse (patho)physiological responses, including cell migration, embryonic development and tissue organization. ${ }^{1}$ Wnt5a, a prototype Wnt, is involved in the inflammatory response. ${ }^{2}$ Wnt5a is reported to stimulate the production of several important inflammatory cytokines and chemokines, such as tumor necrosis factor- $\alpha$, interleukin-6, CCL2, CCL5 and CXCL1, in human endothelial cells, monocytes and bone marrow stromal cells. ${ }^{3-5}$ Wnt5a binds to several members of the Frizzled receptor family, including FZD2, 5 and $8 .^{6}$ The signaling pathways induced by Wnt5a are mediated by canonical ( $\beta$-catennin-dependent) or noncanonical ( $\beta$-catenin-independent) pathways. ${ }^{1,6}$ Of these two signaling pathways, the noncanonical pathway is known to have a function in the inflammatory response induced by Wnt5a. ${ }^{6}$

Neutrophils, which are first recruited to an infected area or injured site by a chemoattractant gradient, are important in the regulation of the immune response, especially the innate immune response. ${ }^{78}$ Various extracellular stimuli can activate neutrophils, resulting in the production of several important mediators: inflammatory cytokines and reactive oxygen species. ${ }^{9,10}$ It is critical for the host defense mechanism that circulating neutrophils are recruited into an infected or injured area. This recruitment of neutrophils can be induced by various chemoattractants. ${ }^{11}$ Chemoattractants, including several chemokines that regulate the activities of neutrophils, have received much attention over the last decade. The chemoattractants bind to specific cell-surface receptors which are G-protein coupled receptors and pertussis toxin (PTX)sensitive. $^{12-14}$ Activation of the receptors elicits intracellular $\mathrm{Ca}^{2+}$ mobilization, cell migration, secretion and production of reactive oxygen species and/or bioactive lipid mediators. ${ }^{12-14}$ Even though neutrophils perform key functions in innate immunity, the effect of Wnt5a on neutrophil activity has not been studied. Here we investigated whether human neutrophils express Wnt5a receptors, and also the function of Wnt5a in neutrophil activity.

${ }^{1}$ Department of Biological Science, Sungkyunkwan University, Suwon, South Korea; ${ }^{2}$ Mitochondria Hub Regulation Center, Dong-A University, Busan, South Korea; ${ }^{3}$ Department of Hematology-Oncology, Ajou University School of Medicine, Suwon, South Korea; ${ }^{4}$ School of Nano-Biotechnology and Chemical Engineering, Ulsan National Institute of Science and Technology, Ulsan, South Korea and ${ }^{5}$ Samsung Advanced Institute for Health Sciences and Technology, Sungkyunkwan University, Seoul, South Korea.

Correspondence: Professor Y-S Bae, Department of Biological Science, Sungkyunkwan University, 300 Cheoncheon-dong, Jangan-gu, Suwon 440-746, South Korea.

E-mail: yoesik@skku.edu

Received 2 December 2012; revised 25 March 2013; accepted 28 March 2013 


\section{MATERIALS AND METHODS}

\section{Materials}

Recombinant mouse Wnt5a protein was from Millipore (Billerica, MA, USA). Recombinant human soluble frizzled related protein-1 (sFRP1) and anti-Wnt5a antibody were obtained from R\&D Systems (Minneapolis, MN, USA). Peripheral blood mononuclear cell separation medium (Histopaque-1077) and cytochrome c were from Sigma (St Louis, MO, USA). PTX, PD98059, SB203580, SP600125, U-73122, U-73343 and LY294002 were from Calbiochem (San Diego, CA, USA). BAY11-7082 was from Enzo Life Sciences (Farmingdale, NY, USA).

\section{Isolation of human neutrophils and monocytes}

This study was approved by the Institutional Review Board of the Ajou University Hospital specifically (approval ID: AJIRB-MED-SMP10-074). We also obtained written informed consent from all participants involved in this study. Human neutrophils and monocytes were isolated as described previously. ${ }^{15}$

\section{RT-PCR Analysis}

Semiquantitative RT-PCR was performed as described previously. The sequences of the primer used were as follows; fzd2: forward, 5'-GCGTCTTCTCCGTGCTCTAC-3'; reverse, 5'-CTGTTGGTGAGG CGAGTGTA-3'. fzd3: forward, 5'-TGAGTGTTCGAAGCTCATGGA-3'; reverse, $5^{\prime}$-ATCACGCACATGCAGAAAAG-3'. fzd4: forward, 5'-AACC TCGGCTACAACGTGAC- ${ }^{\prime}$; reverse, $5^{\prime}$-GTTGTGGTCGTTCTGTGG TG-3'. fzd5: forward, 5'-TGCTACCAGCCGTCCTTCAGT-3'; reverse, $5^{\prime}$-CCATGCCGAAGAAGTAGACCAG-3'. fzd8: forward, 5' - TCTTGT CGCTCACATGGTTC-3'; reverse, 5' -TGTAGAGCACGGTGAACAGG- $3^{\prime}$. GAPDH: forward, 5' ${ }^{\prime}$-GACATCAAGAAGGTGGTGAA-3', reverse, 5'-TGTCATACCAGGAAATGAGC-3'.

\section{Chemotaxis assay}

Chemotaxis assays were performed using multi-well chambers (Neuroprobe, Gaithersburg, MD, USA) as described previously. ${ }^{16}$ Briefly, isolated fresh human neutrophils $\left(25 \mu \mathrm{l}\right.$ of $1 \times 10^{6}$ cells per ml) were placed onto the upper well a $3-\mu \mathrm{m}$ polyhydrocarbon filter. Various concentrations of Wnt5a were applied into lower well. After incubation for $90 \mathrm{~min}$ migrated cells across the filter were counted as described previously. ${ }^{16}$

\section{Measurement of the formation of inositol phosphates in cells \\ The formation of inositol phosphates was determined as described previously. ${ }^{17}$ Briefly, freshly isolated human neutrophils $\left(5 \times 10^{6}\right.$ cells per ml) were labeled with myo- $\left[{ }^{3} \mathrm{H}\right]$ inositol $\left(1 \mu \mathrm{Ci}\right.$ per $10^{6}$ cells) for $12 \mathrm{~h}$ at $37^{\circ} \mathrm{C}$. After stimulating labeled human neutrophils with several concentrations of Wnt5a, ice-cold 5\% perchloric acid $\left(\mathrm{HClO}_{4}\right)$ was added. Produced total inositol phosphates were separated according to standard procedure describe previously. ${ }^{17}$}

\footnotetext{
Measurement of chemokines

Human neutrophils $\left(2 \times 10^{6}\right.$ cells per $\left.0.2 \mathrm{ml}\right)$ were placed in RPMI 1640 medium containing 5\% FBS in 96-well plates, and were kept in a $5 \% \mathrm{CO}_{2}$ incubator at $37^{\circ} \mathrm{C}$. Neutrophils were incubated with several concentrations of Wnt5a $\left(0.1,0.5\right.$ or $\left.1 \mu \mathrm{g} \mathrm{ml}^{-1}\right)$ for $24 \mathrm{~h}$ in the presence or absence of PTX, or kinase inhibitors (PD98059, SB203580 or SP600125). Cell-free supernatants were collected, centrifuged and measured for CXCL8 or CCL2 production by enzyme-linked immunosorbent assay (CXCL8; BD Biosciences, San Jose, CA, USA, CCL2;
}

eBioscience, Inc., San Diego, CA, USA) according to the manufacturer's instructions.

\section{Measurement of the effect of supernatant from lipopolysaccharide-stimulated macrophages on neutrophil chemotaxis}

Human monocytes were differentiated to macrophages by adding M-CSF $\left(10 \mathrm{ng} \mathrm{ml}^{-1}\right)$ for 3 days. Macrophages were stimulated with lipopolysaccharide (LPS; $1 \mu \mathrm{g} \mathrm{ml}^{-1}$ ) for $24 \mathrm{~h}$ and the supernatant was collected. Isolated human neutrophils were incubated with vehicle or anti-Wnt5a antibody for $30 \mathrm{~min}$, suspended at $1 \times 10^{6}$ cells per $\mathrm{ml}$, and were used as described above for chemotaxis.

\section{Statistical analysis}

Results are expressed as mean \pm s.e. for data obtained from the indicated number of experiments. Statistical significance was determined using the Student's $t$-test.

\section{RESULTS}

Chemotactic effect of Wnt5a on human neutrophils

To examine whether human neutrophils express receptors for Wnt5a, we analyzed mRNA for FZD2, 3, 4, 5 and 8 by semiquantitative reverse-transcription PCR. As shown in Figure 1a, human neutrophils expressed FZD2, FZD5 and FZD8 Wnt5a receptors.

We also investigated the effect of Wnt5a on neutrophil chemotaxis. Wnt5a at $0.01-1 \mu \mathrm{M}$ induced migration of human neutrophils according to concentration-response curves (Figure 1b). This implied that Wnt5a induced chemotaxis in human neutrophils. The chemotactic activity of Wnt5a was comparable to the well-known chemoattractant WKYMVm. ${ }^{18}$ As sFRP1 binds to Wnt5a and blocks the action of Wnt5a to its specific receptors, ${ }^{19}$ we examined the effect of sFRP1 on Wnt5a-induced human neutrophil chemotaxis. As shown in Figure 1c, Wnt5a-stimulated chemotaxis of neutrophil was almost completely inhibited by sFRP1. However, WKYMVm-induced neutrophil chemotaxis was not inhibited by sFRP1 (data not shown). This result indicated that Wnt5a stimulated neutrophil chemotaxis via specific receptors in the FZD family.

\section{Wnt5a-stimulated neutrophil chemotaxis is mediated by phospholipase C activity} As many frizzled receptors, including FZD2, FZD5 and FZD8, have been reported as G-protein-coupled receptors, ${ }^{20}$ and many G-protein-coupled receptors can induce the stimulation of phospholipase C (PLC) upon ligand binding, we tested the effect of Wnt5a on PLC activity in human neutrophils. Stimulation of human neutrophils by several different concentrations of Wnt5a elicited an increase in total inositol phosphate formation. This showed a concentration dependency (Figure 2a). These results indicated that Wnt5a stimulated PLC activity in human neutrophils. As Wnt5a stimulated neutrophil chemotaxis, we examined the role of PLC on Wnt5a-stimulated chemotaxis. Preincubation of human neutrophils with a PLC-selective inhibitor (U-73122) before chemotaxis assay almost completely 

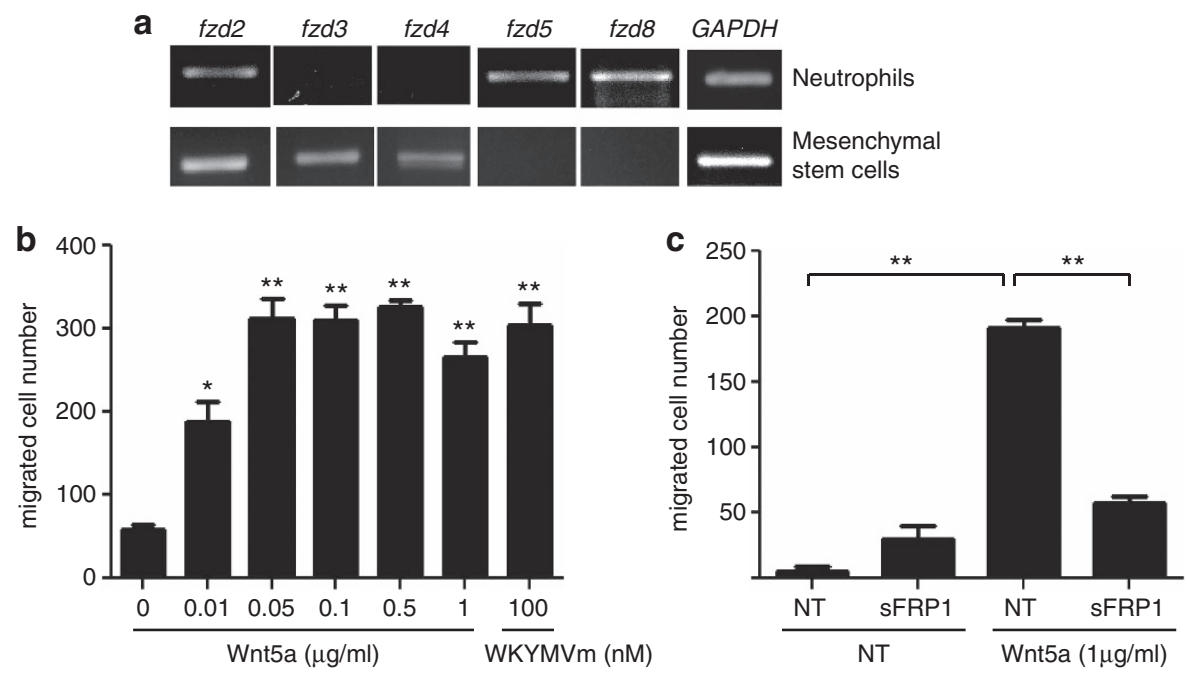

Figure 1 Expression of Wnt5a receptors in human neutrophils and chemotactic activity of Wnt5a on human neutrophils. mRNA was isolated from human neutrophils or human mesenchymal stem cells. Semiquantitative reverse-transcription PCR determined fzd2, fzd3, $\mathrm{fzd} 4$, fzd5 and fzd8 mRNAs. Data are representative of three independent experiments (a). Assays were performed using a modified Boyden chamber assay. Isolated human neutrophils $\left(1 \times 10^{6}\right.$ cells per $\mathrm{ml}$ in serum-free RPMI 1640 medium) were added to the upper wells of a 96-well chemotaxis chamber and migration across a polycarbonate membrane with $3 \mu \mathrm{m}$ pore size was assessed after $1.5 \mathrm{~h}$ at $37^{\circ} \mathrm{C}$ (b). Human neutrophils were incubated with or without $1 \mu \mathrm{gml}-1$ of sFRP1 for $30 \mathrm{~min}$. Cells were used in a chemotaxis assay using $1 \mu \mathrm{g} \mathrm{ml}^{-1}$ of Wnt5a (c). Migrated cells were determined by counting under light microscope. Data are mean $\pm \mathrm{s}$.e.; $n=6$, ${ }^{*} P<0.05$, $* * P<0.01$ versus vehicle treatment.

inhibited Wnt5a-induced neutrophil chemotaxis (Figure 2b). An inactive analog of U-73122, U-73343, did not inhibit Wnt5a-induced chemotaxis. These results indicated that Wnt5a-stimulated neutrophil chemotaxis was mediated by PLC.

Wnt5a-stimulated neutrophil chemotaxis is mediated by phosphoinositide 3-kinase activity

We also tested the effect of Wnt5a on the important signaling molecules mitogen-activated protein kinases (MAPKs) and Akt. Stimulation of human neutrophils with Wnt5a for several different lengths of time caused phosphorylation of ERK, p38 MAPK, JNK and Akt (Figure 3a). Phosphorylation of Akt and ERK was apparent within 2-10 min after Wnt5a stimulation, and phosphorylation of p38 MAPK was sustained from 2 to 30 min after Wnt5a stimulation (Figure 3a). Wn5a transiently stimulated phosphorylation of JNK in human neutrophils at 2 min after stimulation (Figure 3a). ATF2 phosphorylation was also induced by Wnt5a, and its phosphorylation was sustained to $30 \mathrm{~min}$ after stimulation (Figure $3 \mathrm{a}$ ).

We also investigated the role of signaling pathways in the activation of MAPKs and Akt on Wnt5a-stimulated neutrophil chemotaxis, using several inhibitors that are specific for ERK, p38 MAPK, JNK or phosphoinositide 3-kinase (an upstream kinase of Akt). As shown in Figure 3b, Wnt5a-stimulated neutrophil chemotaxis was almost completely inhibited by LY294002 (a phosphoinositide 3-kinase inhibitor), but not by other MAPK inhibitors. These results indicated that the phosphoinositide 3-kinase activity was essential for neutrophil chemotaxis induced by Wnt5a.

\section{Wnt5a stimulates CXCL8 production via ERK and p38 MAPK pathway in human neutrophils}

Human neutrophils produce the important chemokine CXCL8 that recruits several important human leukocytes, including neutrophils. $^{21}$ We measured the effect of Wnt5a on the production of CXCL8 in human neutrophils. Stimulation of human neutrophils with several concentrations of Wnt5a elicited the production of CXCL8 in a concentrationdependent manner, showing maximal activity at $1 \mu \mathrm{g} \mathrm{ml}^{-1}$ Wnt5a (Figure 4a). We also tested the effect of Wnt5a on the production of CXCL8 for various lengths of time. Stimulation of human neutrophils with $1 \mu \mathrm{g} \mathrm{ml}^{-1}$ Wnt5a elicited the production of CXCL8 in a time-dependent manner, with maximal activity at $12 \mathrm{~h}$ after stimulation (Figure 4b). We examined the effect of PTX on the production of CXCL8 in human neutrophils after Wnt5a treatment. CXCL8 production after Wnt5a was not affected by PTX, but rather substantially increased by PTX (Figure 4c). These results indicated that Wnt5a stimulated CXCL8 production from human neutrophils via a PTXinsensitive pathway.

The activation of transcription factors, such as nuclear factor- $\kappa \mathrm{B}(\mathrm{NF}-\kappa \mathrm{B})$ is known to be required for the expression of chemokines, including CXCL8 and CCL2. ${ }^{21,22}$ We investigated the effect of Wnt5a on NF- $\mathrm{KB}$ activation, and 

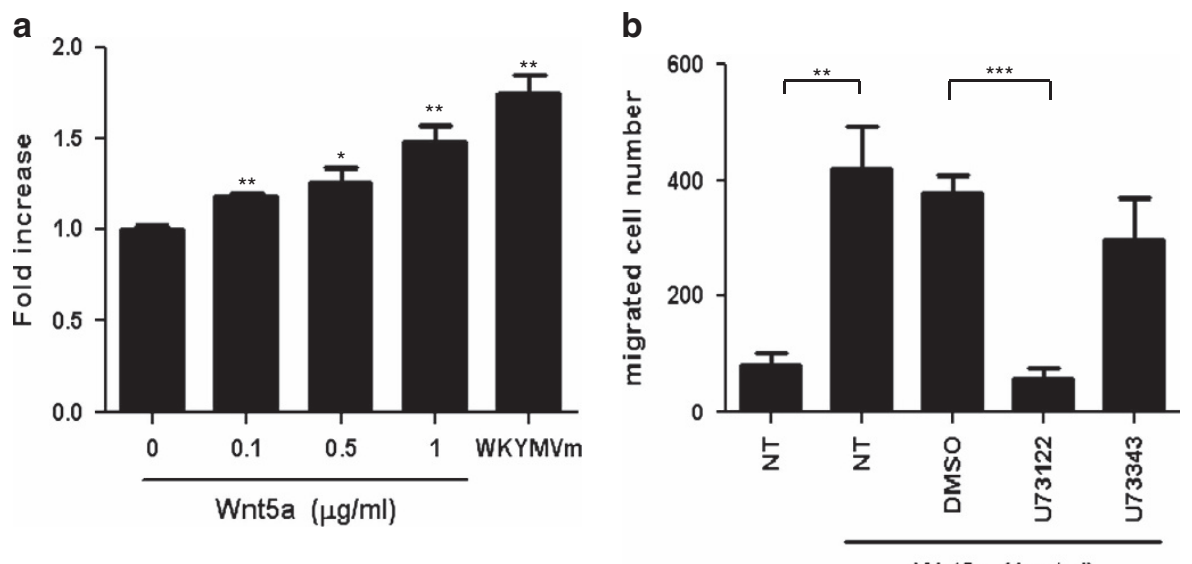

Figure 2 Effect of Wnt5a on PLC activity and neutrophil chemotaxis. Human neutrophils were labeled with myo- $\left[{ }^{3} \mathrm{H}\right]$ inositol $(1 \mu \mathrm{Ci}$ per $10^{6}$ cells) for $12 \mathrm{~h}$ at $37^{\circ} \mathrm{C}$, and were treated with various concentrations of Wnt5a $(0,0.1,0.5$ and $1 \mu \mathrm{m})$ or WKYMVm ( $\left.1 \mu \mathrm{m}\right)$. Total inositol phosphates were eluted with a solution containing $1 \mathrm{~m}$ ammonium formate and $0.1 \mathrm{~m}$ formic acid. $\left[{ }^{3} \mathrm{H}\right]$-inositol phosphates were counted in a scintillation counter (a). Human neutrophils were incubated with or without U-73122 (5 $\mu \mathrm{m})$ or U-73343 (5 $\mu \mathrm{m})$ for $15 \mathrm{~min}$. Cells were used for a chemotaxis assay using $1 \mu \mathrm{gml}-1$ of Wnt5a (b). Migrated cells were determined by counting under light microscope. Data are mean \pm s.e.; $n=6$, ${ }^{*} P<0.05$, ${ }^{*} P<0.01,{ }^{*} * P<0.001$ versus vehicle treatment.
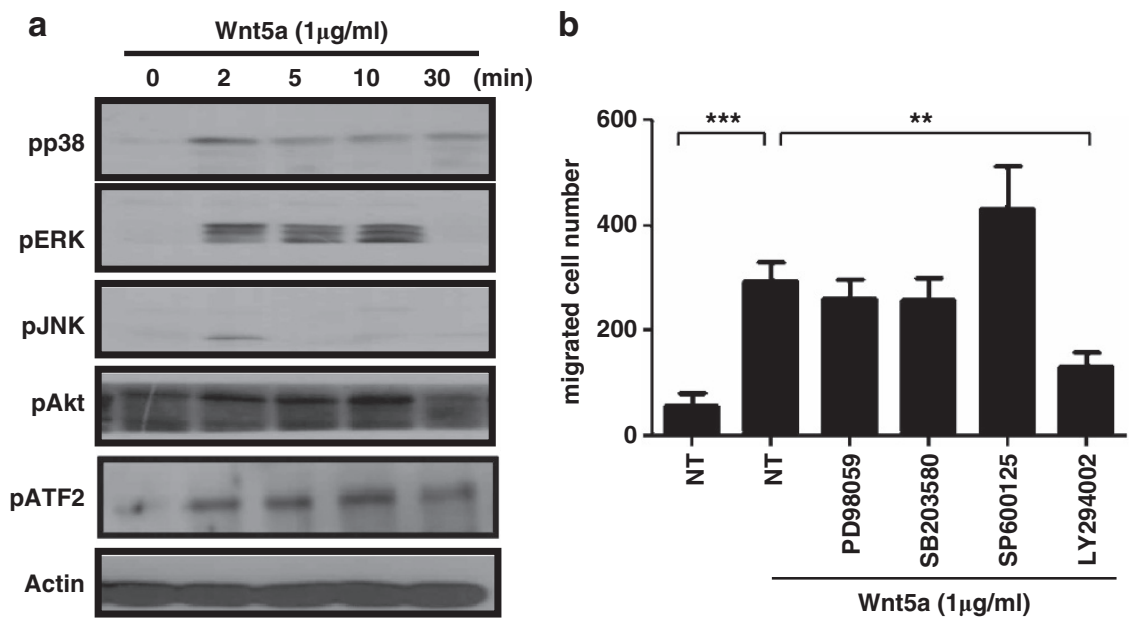

Figure 3 Effect of Wnt5a on MAPKs, Akt and ATF2 activity, and neutrophil chemotaxis. Isolated human neutrophils were stimulated with $1 \mu \mathrm{g} \mathrm{ml} \mathrm{m}^{-1}$ Wnt5a for $0,2,5,10$ or $30 \mathrm{~min}$, then $30 \mu \mathrm{g}$ of cell lysates were subjected to SDS-polyacrylamide gel electrophoresis, followed by immunoblot analysis using anti-phospho-ERK, anti-phospho-p38 MAPK, anti-phospho-JNK, anti-phospho-Akt or anti-phospho-ATF2. Western blots were performed with anti-actin to confirm sample loading. Data are representative of three independent experiments (a).

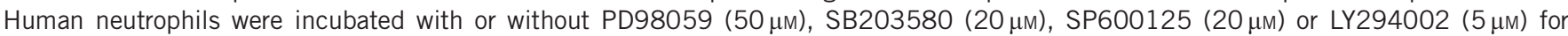
$15 \mathrm{~min}$ (60 min for PD98059). Cells were used for chemotaxis assays using $1 \mu \mathrm{g} \mathrm{ml}-1$ of Wnt5a (b). Migrated cells were determined by counting under light microscope. Data are mean \pm s.e.; $n=5,{ }^{* *} P<0.01,{ }^{* * *} P<0.001$.

also tested the role of MAPK pathways on Wnt5a-stimulated CXCL8 production in human neutrophils. Wnt5a-induced CXCL8 production was inhibited by SB203580 (a p38 MAPK inhibitor), but not by PD98059 (an ERK pathway inhibitor), indicating that p38 MAPK activity was involved in Wnt5astimulated CXCL8 production. Preincubation of neutrophils with SP600125 (a JNK inhibitor) before Wnt5a stimulation elicited increased production of CXCL8 (Figure 4d). We also tested the role of NF- $\kappa B$ on the production of CXCL8 induced by Wnt5a, using an NF-אB-selective inhibitor (BAY11-7082). Preincubation of human neutrophils with BAY11-7082 before addition of Wnt5a markedly increased Wnt5a-induced CXCL8 production (Figure 4d).

Wnt5a-stimulated CCL2 production is mediated by a PTXsensitive G-protein, JNK and NF- $\kappa B$ in human neutrophils Another important chemokine, CCL2, recruits some leukocytes, including monocytes, and is produced by human 
a
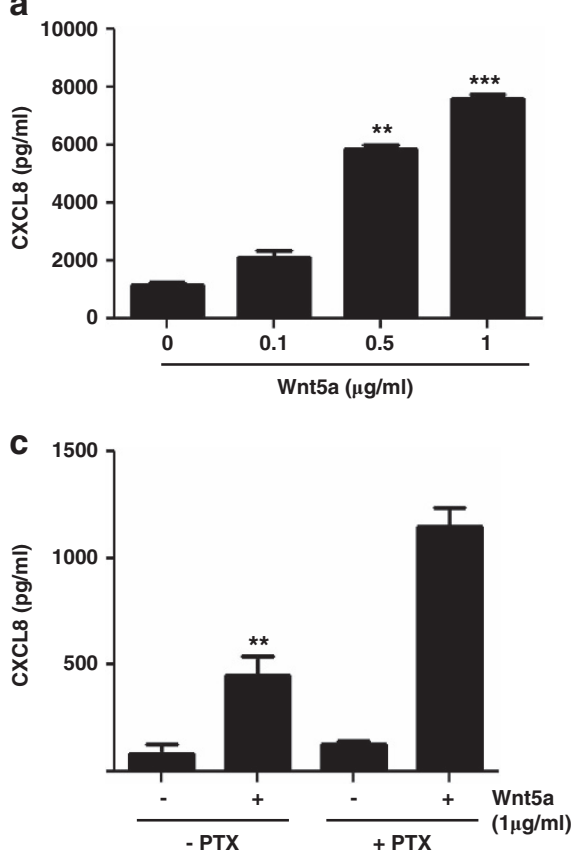

b

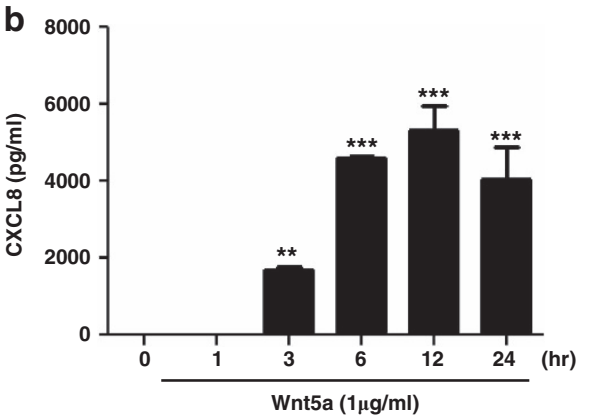

d

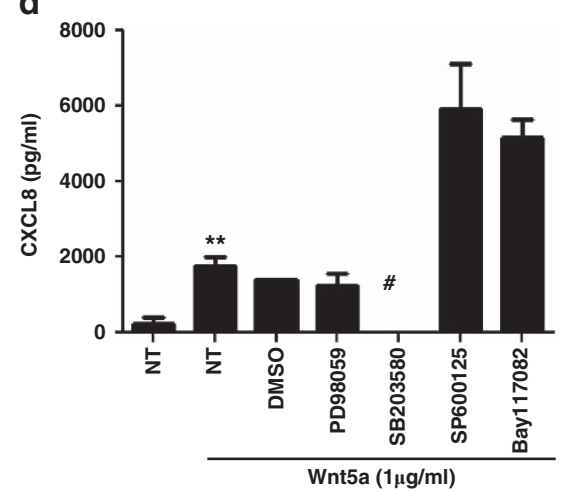

Figure 4 Effect of Wnt5a on CXCL8 production in human neutrophils. Isolated human neutrophils were stimulated with several concentrations of Wnt5a for $24 \mathrm{~h}$ (a) or with $1 \mu \mathrm{g} \mathrm{ml} \mathrm{l}^{-1}$ of Wnt5a for different times (b). Neutrophils were preincubated with or without $1 \mu \mathrm{g} \mathrm{ml}^{-1}$ of PTX for $4 \mathrm{~h}$ then stimulated with $1 \mu \mathrm{gml}-1$ of Wnt5a for $24 \mathrm{~h}$ (c). Human neutrophils were incubated with or without

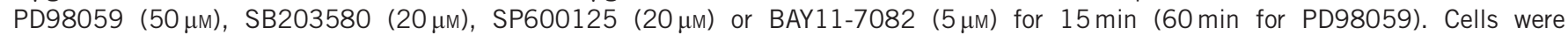
stimulated with $1 \mu \mathrm{g} \mathrm{ml}^{-1}$ of Wnt5a for $24 \mathrm{~h}(\mathbf{d})$. Supernatants were collected from cultures and evaluated for CXCL8 (a-d) production by enzyme-linked immunosorbent assay. Data are means \pm s.e.; $n=6,{ }^{* *} P<0.01,{ }^{* * *} P<0.001$ versus vehicle treatment; ${ }^{\#} P<0.05$ versus dimethylsulfoxide plus Wnt5a-treated control.

neutrophils in response to extracellular stimuli. ${ }^{23}$ We examined the effect of Wnt5a on the production of CCL2 in human neutrophils. Stimulation of human neutrophils with several concentrations of Wnt5a elicited the production of CCL2 in a concentration-dependent manner, showing maximal activity at $1 \mu \mathrm{g} \mathrm{ml}^{-1}$ Wnt5a (Figure 5a). We also tested the effect of Wnt5a on the production of CCL2 for various lengths of time. Stimulation of human neutrophils with $1 \mu \mathrm{g} \mathrm{ml}^{-1}$ Wnt5a elicited the production of CCL2 in a time-dependent manner, with maximal activity at $12 \mathrm{~h}$ after stimulation (Figure 5b). For PTX sensitivity, CCL2 production by Wnt5a was completely affected by PTX (Figure 5c). These results indicated that Wnt5a stimulated CCL2 production from human neutrophils via PTX-sensitive pathway. We also tested the function of several MAPK pathways on Wnt5a-stimulated CCL2 production in human neutrophils. Wnt5a-induced CCL2 production was inhibited by SB203580 (a p38 MAPK inhibitor), PD98059 (an ERK pathway inhibitor) and SP600125 (a JNK inhibitor), indicating that all the three MAPK activities were involved in Wnt5a-stimulated CCL2 production. We also tested the role of NF- $\mathrm{KB}$ on the production of CXCL8 induced by Wnt5a using the NF-אB-selective inhibitor BAY11-7082. Preincubation of human neutrophils with BAY11-7082 before addition of Wnt5a strongly inhibited Wnt5a-induced CCL2 production.

Macrophage conditioned medium stimulated with LPS induces neutrophil chemotactic migration

According to previous reports, Wnt5a is produced mainly by stimulated macrophages. ${ }^{2,3}$ Activated macrophages secrete various soluble factors, which in turn induce migration of other leukocytes, including through several chemoattractants. $^{24}$ We demonstrated that Wnt5a stimulated human neutrophil chemotactic migration (Figure 1). Previously it has been reported that activation of macrophages by LPS enhances the expression of Wnt5a in mRNA and protein levels. $^{25}$ To test the hypothesis that LPS stimulated macrophages to produce Wnt5a-mediating neutrophil chemotaxis, we examined the effect of chemotactic migration by neutrophils induced by supernatant from LPS-stimulated macrophages. Neutrophil chemotaxis was strongly induced by LPS-stimulated macrophage supernatant (Figure 6). This was significantly inhibited by anti-Wnt5a antibody. 

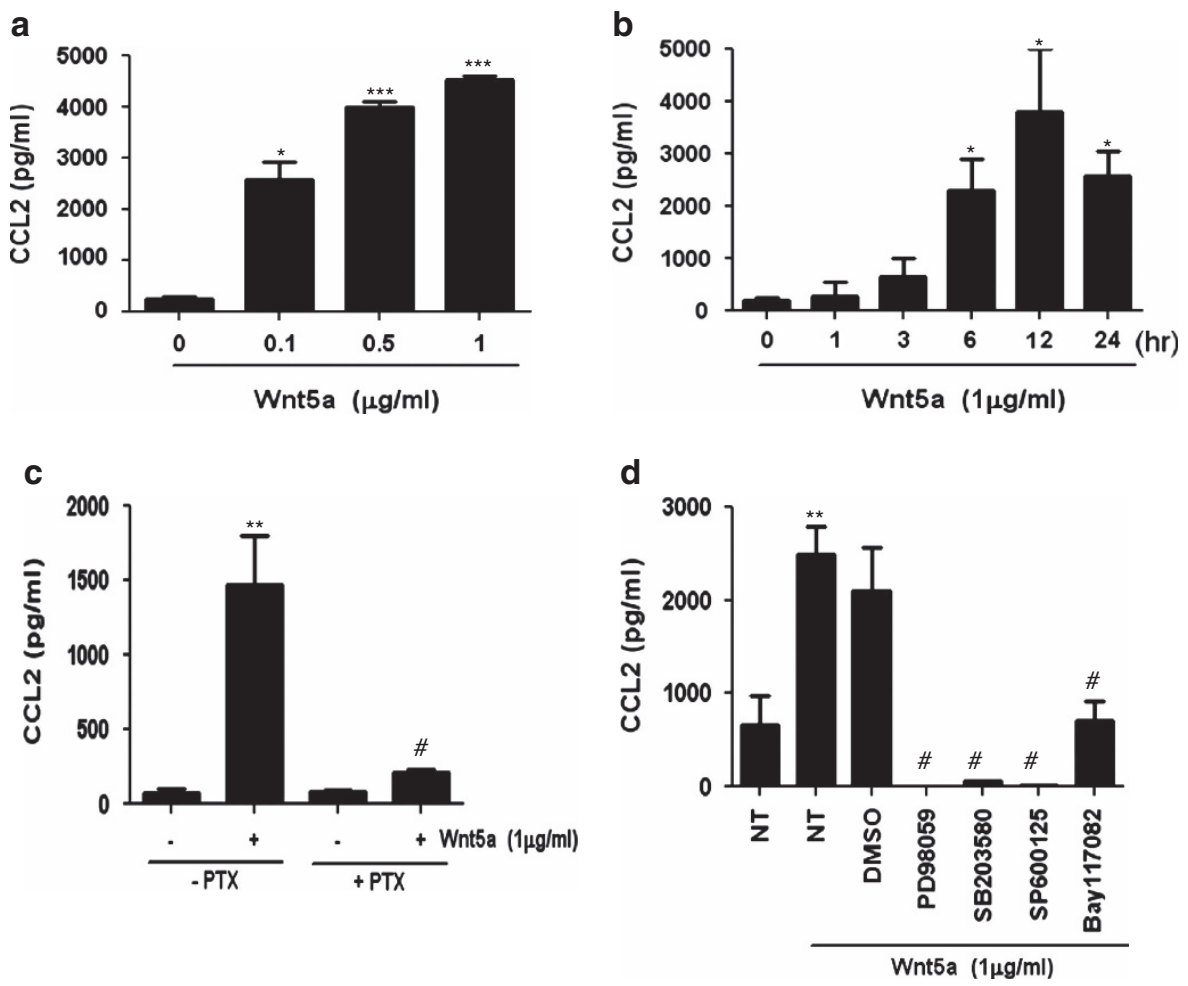

Figure 5 Effect of Wnt5a on CCL2 production from human neutrophils. Isolated human neutrophils were stimulated with several concentrations of Wnt5a for $24 \mathrm{~h}$ (a) or with $1 \mu \mathrm{g} \mathrm{ml}-1$ of Wnt5a for different times (b). Neutrophils were preincubated with or without $1 \mu \mathrm{g} \mathrm{ml}^{-1}$ of PTX for $4 \mathrm{~h}$ and stimulated with $1 \mu \mathrm{g} \mathrm{ml}-1$ of Wnt5a for $24 \mathrm{~h}$ (c). Human neutrophils were incubated with or without

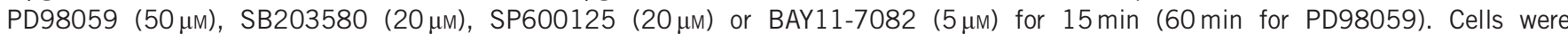
stimulated with $1 \mathrm{mg} \mathrm{ml}^{-1}$ of Wnt5a for $24 \mathrm{~h}$ (d). Supernatants were collected from cultures and were evaluated for CCL2 (a-d) production by enzyme-linked immunosorbent assay. Data are means \pm s.e.; $n=6,{ }^{*} P<0.05,{ }^{* *} P<0.01,{ }^{* *} P<0.001$ versus vehicle treatment; ${ }^{\#} P<0.05$ versus dimethylsulfoxide plus Wnt5a-treated control.

We confirmed that anti-Wnt5a antibody strongly inhibited Wnt5a-stimulated neutrophil chemotaxis (data not shown). We also tested direct effect of LPS on neutrophil chemotaxis; LPS itself could not induce neutrophil chemotaxis (data not shown).

\section{DISCUSSION}

As neutrophils are important in human early defense activity, ${ }^{7-10}$ the identification of endogenous molecules that regulate neutrophil function is of paramount importance. In this study we demonstrated that Wnt5a, an endogenous glycoprotein secreted from stimulated macrophages, stimulated neutrophil activity. Functionally, we found that Wnt5a stimulated neutrophil chemotaxis and chemokine production. However, Wnt5a failed to stimulate superoxide anion production from human neutrophils (data not shown). Since superoxide anion production is required for killing invading pathogens, Wnt5a might not stimulate the bactericidal activity of neutrophils against pathogens. As Wnt5a stimulated the production of two important chemokines, CXCL8 and CCL2, which are potent chemoattractants for neutrophils and monocytes, Wnt5a might regulate the immune response by inducing leukocyte migration.

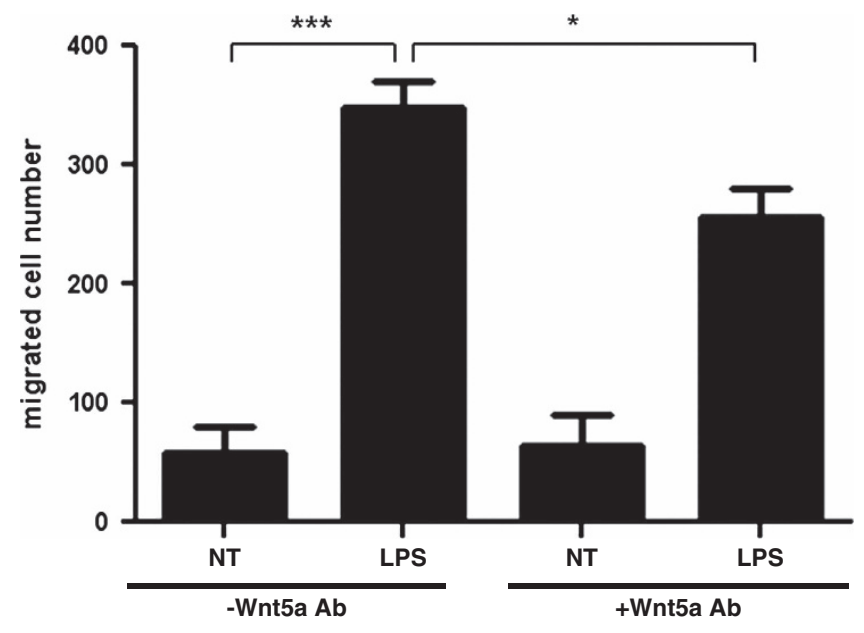

Figure 6 Role of Wnt5a on the chemotactic migration of neutrophils induced by supernatant from LPS-stimulated macrophages. Human macrophages were stimulated with LPS $(1 \mu \mathrm{g} \mathrm{ml}-1)$ for $24 \mathrm{~h}$, and supernatant was collected. Freshly isolated human neutrophils were used for a chemotaxis assay using supernatant from LPS-stimulated macrophages in the absence or presence of anti-Wnt5a antibody $\left(2 \mu \mathrm{gll}^{-1}\right)$ for $1.5 \mathrm{~h}$. Migrated cells were determined by counting under light microscope. Data are mean \pm s.e.; $n=4,{ }^{*} P<0.05,{ }^{* *} P<0.001$. 
We demonstrated that Wnt5a stimulated the production of two important chemokines, CXCL8 and CCL2 (Figures 4, 5). To identify the signaling pathways involved in the Wnt5astimulated chemokine production, we found that production of CCL2 induced by Wnt5a, but not by CXCL8, was completely inhibited by PTX (Figures 4, 5). These results indicated that a PTX-sensitive G-protein-mediated pathway was necessary for Wnt5a-stimulated production of CCL2, but not CXCL8. As FZD2, a receptor for Wnt5a, signaling is known to be mediated by PTX-sensitive G-proteins, ${ }^{26}$ our results support the hypothesis that Wnt5a may stimulate FZD2, which is coupled to PTX-sensitive G-proteins, thus resulting in CCL2 expression. In addition, JNK and NF- $\mathrm{KB}$ activities were required for Wnt5a-stimulated production of CCL2 but not CXCL8. Taken together, our results suggested that Wnt5a stimulated CCL2 via a PTX-sensitive G-protein, JNK and the NF- $\mathrm{KB}$ pathway. However, Wnt5a-stimulated CXCL8 production used a signaling pathway independent of a PTX-sensitive G-protein, JNK and NF- $\kappa B$. As the Wnt5a-induced CXCL8 production was mediated by p38 MAPK (Figure 4d), which stimulates ATF-2 activity, ATF-2 activity may be involved in Wnt5ainduced CXCL8 production.

The activation of macrophages by LPS has been known to enhance the expression of Wnt $5 \mathrm{a}^{25}$. To understand the functional role of Wnt5a in vivo, we examined the effect of supernatant from LPS-stimulated macrophages on neutrophil chemotaxis. We found that the effect of supernatant collected from macrophages stimulated with LPS was significantly inhibited by anti-Wnt5a antibody (Figure 6). From these results, we suggest that Wnt5a may be secreted from activated macrophages with LPS and strongly induces neutrophil chemotaxis. In addition, Wnt5a stimulated secretion of CXCL8 and CCL2 (Figures 4, 5), which are potent chemoattactants for neutrophils and monocytes, respectively. ${ }^{27,28}$ Taken together, these results suggested that Wnt5a may mediate an important positive loop for the recruitment of neutrophils, monocytes and macrophages into event area in response to infection.

Previously, Lee and colleagues ${ }^{3}$ demonstrated that Wnt5a stimulates endothelial cells, resulting in the expression of several important inflammatory molecules, such as COX-2, CXCL8, CX3CL1 and E-selectin. In this study we showed that Wnt5a stimulated neutrophils, resulting in chemotactic migration and expression of two important chemokines (CXCL8 and CCL2). The previous reports and our findings suggested that Wnt5a might induce inflammatory responses by modulating not only endothelial cell activity but also neutrophil activity. A $\beta$-catenin-independent signaling pathway mediated this Wnt5a-induced inflammatory response in neutrophils as well as in endothelial cells.

\section{ACKNOWLEDGEMENTS}

This paper was supported by the Samsung Research Fund, Sungkyunkwan University, 2011, and by High Seoul Science (Humanities) Fellowship funded by Seoul Scholarship Foundation.
1 Clevers H, Nusse R. Wnt/ß-catenin signaling and disease. Cell 2012; 149: 1192-1205.

2 Pereira CP, Bachli EB, Schoedon G. The wnt pathway: a macrophage effector molecule that triggers inflammation. Curr Atheroscler Rep 2009; 11: 236-242.

3 Kim J, Kim J, Kim DW, Ha Y, Ihm MH, Kim H, Song K, Lee I. Wnt5a induces endothelial inflammation via beta-catenin-independent signaling. J Immunol 2010; 185: 1274-1282.

4 Kim J, Chang W, Jung Y, Song K, Lee I. Wnt5a activates THP-1 monocytic cells via a $\beta$-catenin-independent pathway involving JNK and NF-KB activation. Cytokine 2012; 60: 242-248.

5 Rauner M, Stein N, Winzer M, Goettsch C, Zwerina J, Schett G et al. WNT5A is induced by inflammatory mediators in bone marrow stromal cells and regulates cytokine and chemokine production. J Bone Miner Res 2012; 27: 575-585.

6 Kikuchi A, Yamamoto H, Sato A, Matsumoto S. Wnt5a: its signalling, functions and implication in diseases. Acta Physiol (Oxf) 2012; 204: 17-33.

7 Williams MR, Azcutia V, Newton G, Alcaide P, Luscinskas FW. Emerging mechanisms of neutrophil recruitment across endothelium. Trends Immunol 2011; 32: 461-469.

8 Kobayashi SD, DeLeo FR. Role of neutrophils in innate immunity: a systems biology-level approach. Wiley Interdiscip Rev Syst Biol Med 2009; 1: 309-333.

9 Sadik CD, Luster AD. Lipid-cytokine-chemokine cascades orchestrate leukocyte recruitment in inflammation. J Leukoc Biol 2012; 91: 207-215.

10 Robinson JM. Phagocytic leukocytes and reactive oxygen species. Histochem Cell Biol 2009; 131: 465-469.

11 Sanz MJ, Kubes P. Neutrophil-active chemokines in in vivo imaging of neutrophil trafficking. Eur J Immunol 2012; 42: 278-283.

12 Rittner HL, Labuz D, Schaefer M, Mousa SA, Schulz S, Schäfer M et al. Pain control by CXCR2 ligands through $\mathrm{Ca} 2+$-regulated release of opioid peptides from polymorphonuclear cells. FASEB J 2006; 20: 2627-2629.

13 Mahadeo DC, Janka-Junttila M, Smoot RL, Roselova P, Parent CA. A chemoattractant-mediated Gi-coupled pathway activates adenylyl cyclase in human neutrophils. Mol Biol Cell 2007; 18: 512-522.

14 Bae YS, Park EY, Kim Y, He R, Ye RD, Kwak JY et al. Novel chemoattractant peptides for human leukocytes. Biochem Pharmacol 2003; 66: 1841-1851

15 Bae YS, Bae H, Kim Y, Lee TG, Suh PG, Ryu SH. Identification of novel chemoattractant peptides for human leukocytes. Blood 2001; 97: 2854-2862.

16 Bae GH, Lee HY, Jung YS, Shim JW, Kim SD, Baek SH et al. Identification of novel peptides that stimulate human neutrophils. Exp Mol Med 2012; 44: 130-137.

17 Baek SH, Seo JK, Chae CB, Suh PG, Ryu SH. Identification of the peptides that stimulate the phosphoinositide hydrolysis in lymphocyte cell lines from peptide libraries. J Biol Chem 1996; 271: 8170-8175.

18 Bae YS, Kim Y, Kim Y, Kim JH, Suh PG, Ryu SH. Trp-Lys-Tyr-Met-Val-DMet is a chemoattractant for human phagocytic cells. J Leukoc Biol 1999; 66: 915-922.

19 Goodwin AM, D'Amore PA. Wnt signaling in the vasculature. Angiogenesis 2002; 5: 1-9.

20 Schulte G. International Union of Basic and Clinical Pharmacology. LXXX. The class Frizzled receptors. Pharmacol Rev 2010; 62: 632-667.

21 Cao J, Gong Y, Yin Y, Wang L, Ying B, Chen T, Zhang X. Pneumococcal proteins PspA and PspC induce CXCL 8 production in human neutrophils: implications in pneumococcal infections. Microbes Infect 2010; 12: 1051-1060

22 Matsuyama W, Wang L, Farrar WL, Faure M, Yoshimura T. Activation of discoidin domain receptor 1 isoform $b$ with collagen up-regulates chemokine production in human macrophages: role of p38 mitogenactivated protein kinase and NF-kappa B. J Immunol 2004; 172: 2332-2340.

23 Nijnik A, Madera L, Ma S, Waldbrook M, Elliott MR, Easton DM et al. Synthetic cationic peptide IDR-1002 provides protection against bacterial infections through chemokine induction and enhanced leukocyte recruitment. J Immunol 2010; 184: 2539-5250.

24 Goodman RB, Forstrom JW, Osborn SG, Chi EY, Martin TR. Identification of two neutrophil chemotactic peptides produced by porcine alveolar macrophages. J Biol Chem 1991; 266: 8455-8463. 
25 Zhao C, Ma H, Bu X, Wang W, Zhang N. SFRP5 inhibits gastric epithelia cell migration induced by macrophage-derived Wnt5a. Carcinogenesis 2013; 34: 146-152.

26 Ahumada A, Slusarski DC, Liu X, Moon RT, Malbon CC, Wang HY. Signaling of rat Frizzled-2 through phosphodiesterase and cyclic GMP. Science 2002; 298: 2006-2010.

27 Murphy PM, Baggiolini M, Charo IF, Hebert CA, Horuk R, Matsushima K, Miller LH et al. International Union of Pharmacology: XXII. Nomenclature for chemokine receptors. Pharmacol Rev 2000; 52: $145-176$.
28 Deshmane SL, Kremlev S, Amini S, Sawaya BE. Monocyte chemoattractant protein-1 (MCP-1): an overview. J Interferon Cytokine Res 2009; 29: 313-326.

(c) (1) (-) $\Theta$ This work is licensed under a Creative Commons Attribution-NonCommercial-NoDerivs 3.0 Unported License. To view a copy of this license, visit http:// creativecommons.org/licenses/by-nc-nd/3.0/ 